\title{
Designing the Social Internet of Things
}

\begin{tabular}{|c|c|}
\hline Alessandro Soro & Tuck W Leong \\
\hline Margot Brereton & University of Technology Sydney \\
\hline Paul Roe & (NSW) Australia \\
\hline \multicolumn{2}{|l|}{ Peta Wyeth } \\
\hline Daniel Johnson & Wendy Ju \\
\hline Aloha Hufana Ambe & Stanford University, USA \\
\hline Queensland University of & \\
\hline Technology & Silvia Lindtner \\
\hline Brisbane (QLD) Australia & University of Michigan, USA \\
\hline Ann Morrison & Yvonne Rogers \\
\hline Aalborg University, Denmark & University College London, UK \\
\hline Shaowen & Jacob Buur \\
\hline Indiana University, USA & University of Southern Denmark \\
\hline
\end{tabular}

Permission to make digital or hard copies of part or all of this work for personal or classroom use is granted without fee provided that copies are not made or distributed for profit or commercial advantage and that copies bear this notice and the full citation on the first page. Copyrights for thirdparty components of this work must be honored. For all other uses, contact the Owner/Author.

Copyright is held by the owner/author(s).

CHI'17 Extended Abstracts, May 06-11, 2017, Denver, CO, USA

.

http://dx.doi.org/10.1145/3027063.3027069

\begin{abstract}
What role do people have in the Internet of Things? Compared to the impressive body of research that is currently tackling the technical issues of the Internet of Things, social aspects of agency, engagement, participation, and ethics, are receiving less attention. The goal of this 'Designing the Social Internet of Things' workshop is to contribute by shedding light on these aspects. We invite prospective participants to take a humanistic standpoint, explore people's relations with 'things' first, and then build on such relations so as to support socially relevant goals of engagement, relatedness, participation, and creativity.
\end{abstract}

\section{Author Keywords}

Social Internet of Things; smart objects; engagement; relatedness; participation; creativity.

\section{ACM Classification Keywords}

H5.m. Information interfaces and presentation (e.g., $\mathrm{HCI}$ ): Miscellaneous.

\section{Background}

What role do people have in the Internet of Things?

The recent explosion of interest surrounding the Internet of Things, both from industry and academia, appears to focus mainly on the technical issues of networking, communication, sensing and reasoning. The well-known framework by Atzori, Iera and Morabito [1] identified several 'visions' that characterise the 
Internet of Things: 'things' oriented, 'Internet' oriented, and 'semantic' oriented. Here the lack of a 'humans' oriented vision is indicative of a lack of attention, at least in the initial phases of the IoT conceptualisation, from the HCI community.

A narrow view of objects as commodities to 'smartify' risks smothering the great opportunities for design that objects offer in terms for example, of fostering independence, supporting creativity, engaging in meaningful relations (see e.g. [8]).

More recently, many authors have called for bringing people back into the IoT loop (e.g. $[3,6,8,10,11])$. Things are recognized as having a larger role than as acting as endpoints within a technical infrastructure. The things contribute with their own meaning, their own thingness, as well as becoming meaningful through the actions and interactions of humans, to act with and through [5]. In these alternative visions, 'social objects' encompass the intricate relations between things, people, and environments [7], that can hardly be captured from a technology-only oriented perspective [9] without incurring the risk to reduce the person to a passive subject of the 'smart' technology.

This body of research is showing that building on people's goals, values and attachment to objects can inform the design of the IoT, and at the same time open up opportunities for approaching bigger concerns over ethics, environmental consequences (anthropocene), and the politics of IoT.

People value objects for what they represent, for what they allow to achieve, or as symbols of a relationship [11]. People's homes (often the most expensive and stable 'thing' one possess) are the center of affective lives [6]. People value the routines and habits that objects within those homes support, and they will often resist change if those routines are menaced [4]. By understanding such routines [2] and, in combination with concepts of appropriation and design after design, we can rethink smart objects as those that better fit and bend to people's values and habits [10]. The question of how the relationship between people and objects has shifted, or can shift as a consequence of objects becoming smarter and somewhat autonomous, is however entirely open.

This workshop aims at uncovering these opportunities by inviting researchers and practitioners to reflect on the internet of things from a humanistic standpoint.

It will continue the conversation started at OzCHI 2014 with the workshop 'Social Internet of Things', in which we explored questions such as: why are internet enabled objects struggling to emerge as consumer devices? What can we learn from success (and failure) stories? Who are smart objects for, what goals do they serve and what skills are required to build, use and maintain them?

There, participants contributed their different takes of the 'social' factor, and how this affected their vision for an Internet of Things as, for example, shared or sharable, designed to foster connectedness, or even aimed at public good and social justice.

A follow up workshop 'Social Internet of Things: the Challenges beyond the Utopia' accepted at OzCHI 2016 is currently accepting submissions and seeks critical and original positions about the current research on 
smart objects and internet of things, particularly when directed at children, older adults, or vulnerable users.

These previous editions focused on the practice of IoT design rather than on the theoretical underpinning of people's relationship with things. To move a further step we invite contributions by prospective participants, both on the theory and practice of conceptualizing the Social Internet of Things.

\section{Theory...}

- User centred design, participatory design and design for appropriation of Internet of Things applications and devices

- Socio-material assemblages, actor networks and agency in the IoT

- Feminist, ethical, and critical perspectives on the IoT

- Phenomenological accounts of interactions with objects

\section{... and Practice}

- Internet of Things for socially relevant goals: e.g. afford independence, provide comfort, communicate prestige, preserve tradition, maintain social relation, foster creativity;

- Unique objects for unique needs: e.g. IoT for communities of professionals, ageing people, students, users with disabilities;

- Privacy issues/controversial experience reports from IoT scenarios, reflections on the environmental footprint of the IoT

- Socialization around the Internet of Things: toolkits and practices for communities of makers;

\section{Organizers}

Alessandro Soro is a postdoctoral research fellow at Queensland University of Technology. His research is focused on natural interaction, including design to support social interaction and natural interfaces for special contexts, such as smart cars and interactive spaces. He is co-author of $30+$ research papers and coeditor of 6 collective works gathering workshops/conference proceedings.

Margot Brereton is a professor at QUT where she leads the computer human interaction discipline and researches the participatory interaction design of ubiquitous computing technologies and their interfaces. She develops innovative designs, methods, and theoretical understandings by designing to support real user communities in selected challenging contexts. Her approach is highly iterative and often involves growing user communities as the design evolves, by understanding and responding to socio-cultural factors.

Paul Roe is a full professor in the Science and Engineering Faculty at QUT, in Brisbane Australia. His research concerns how technology can benefit environment and community, particularly for new kinds of environmental monitoring and novel interfaces which promote community access and engagement. He researches, designs, builds and evaluates novel computer systems.

Peta Wyeth is an Associate Professor at Queensland University of Technology and is at the forefront of research into emerging technology for games and other interactive experiences. She has wide-ranging experience in the application of human-computer interaction and interaction design techniques for the 
development of technology for education and entertainment. She builds intelligent, ubiquitous technology that children and adults can use in meaningful, engaging and appropriate ways

Daniel Johnson leads the QUT Games Research and Interaction Design Lab and is an Associate Professor in the Bachelor of Games and Interactive Entertainment. His research interests include motivations for videogame play, the player experience, the impact of videogames on wellbeing, and gamification. Over the past decade, Daniel has undertaken consultancies exploring usability, user experience and design issues in entertainment and non-leisure software.

Aloha Hufana Ambe is a PhD candidate with the $\mathrm{CHI}$ discipline at QUT. Her research interests are co-design of interactive technologies directed at older people.

Ann Morrison is an Associate Professor for Media Technology Section, Aalborg University, Denmark. Ann leads the Urban Vibrations Lab and designs a range of tangible solutions to enhance states of well-being, mobility, safety and social interaction for everyday circumstance, assistive care and urban environments.

Shaowen Bardzell is an Associate Professor of Informatics in the School of Informatics and Computing at Indiana University. Known for her work in feminist $\mathrm{HCI}$, Bardzell's research explores the contributions of design, feminism, and social science to support technology's role in social change. Recent research foci have included criticality in design, care ethics and feminist utopian perspectives on IT, and culture and creative industries in Asia.
Tuck W Leong is a Senior Lecturer at the University of Technology Sydney. He specialises in human-centred approaches of inquiry and technology design. Tuck's recent research explored Participatory Design approaches to support ageing people to envision a role for the IoT in their everyday lives.

Wendy Ju is an Associate Professor in the Graduate Program in Design at California College of the Arts, and Executive Director of the Center for Design Research at Stanford University. Ju uses a design research approach to investigate human interaction with automation, particularly human-robot interaction and autonomous car interface design.

Silvia Lindtner is an assistant professor at the University of Michigan in the School of Information. She researches, writes and teaches about DIY (do-ityourself) maker culture, with a particular focus on its intersections with manufacturing and industry development in China. Drawing on her background in interaction design and media studies, she merges ethnographic methods with approaches in design and making. This allows her to provide deep insights into emerging cultures of technology production and use, from a sociological and technological perspective.

Yvonne Rogers is a Professor of Interaction Design, the director of UCLIC and a deputy head of the Computer Science department at UCL. Her research interests are in the areas of ubiquitous computing, interaction design and human-computer interaction. A central theme is how to design interactive technologies that can enhance life by augmenting and extending everyday, learning and work activities. This involves informing, building and evaluating novel user 
experiences through creating and assembling a diversity of pervasive technologies.

Jacob Buur is Professor of User-Centred Design at the Mads Clausen Institute for Product Innovation,

University of Southern Denmark, and research director of the strategic research centre SPIRE. With 25 employees, SPIRE aims to establish the theoretical foundation for 'Participatory Innovation' - a new approach to user-driven innovation that expands the notion of user and includes business modeling in the user collaboration. SPIRE is cross-disciplinary, uniting researchers from design-antropology, interaction design, interaction analysis, business, innovation management and SPIRE collaborates with the theatre company Dacapo and Danish and international industries.

\section{Website \& Pre-Workshop plans}

A website with the call for submissions, aims and scope of the workshop, and additional materials will be online short after acceptance. The webpage will be connected to a public Facebook page where all updates will be posted. The hosting website is

http://www.designparticipation. net/2016/11/26/siotchi2017-workshop.htm

The call for participation will be advertised online through $\mathrm{HCI}$ related mailing lists (chi-announcements, pdworld, BCS-HCI, as well as local national HCI lists, e.g. Australian chisigmail, Italian SIGCHI-It, etc).

Prospective participants will be invited to contribute a position paper, introducing their background and interest. If the number of submissions exceeds the 25 recommended maximum size, the organizers will select those ones more likely to contribute a sparkling discussion before, during and after the workshop.

In preparation for the workshop participants will be invited to reflect on people's relationship with 'things' and how those relationships can be inform the design of a human-centred Internet of Things. Reflections based on interviews with third parties or on auto-ethnographic accounts will be both equally welcome. These contributions will be hosted on the Facebook page for everyone's perusal. If the reflection involves one particular 'thing' participants will be invited to bring it along for the workshop (either the real thing or a placeholder, e.g. a toy car for one's real car, pictures for one's home, etc).

\section{Workshop Structure}

The full-day workshop will be organized in four sessions of 1.5 to 2 hours each.

\section{Session 1: welcome and introductions. The} organizers will welcome the participants and introduce themselves, their research interest, and overall motivation and plan for the workshop. They will also briefly summarize the discussion that already took place on the Facebook page, as a starting point for discussion. All participants will then be invited to introduce themselves and present their reflections.

Session 2: working in groups. Participants will be invited to form groups of $4 / 5$ people. The organizers will spread across all groups to work as facilitators.

Participants will be provided with material for sketching and building low fidelity prototypes, and encouraged to build on the reflections contributed by everyone, trying to articulate use scenarios. Participants may also bring 
objects for discussion about how they have been/may be 'smartified'. The goal is to show how the augmentation can concretely afford independence, provide comfort, communicate prestige, preserve tradition, maintain social relation, foster creativity, etc.

Session 3: group presentations. Working in 'plenary' session again, one or two speakers for each group will present the findings, with major emphasis on the people's point of view.

\section{Session 4: wrapping up and lateral thinking. In} this final session participants will be invited to further reflect on the implications of their designs. How do they really empower people and enhance agency? How can possibly these designs affect people's expectations and conceptions of privacy? What is the dark side of these designs, can they possibly retort against their users? What novel forms of participation (e.g. in social life, in family events, in one's community, in politics, in the global economy) may become possible thanks to these designs, and for whom?

\section{Post-Workshop Plans}

The organizers already have an agreement with Springer for an edited book on the theme 'Social Internet of Things' to which all participants will be invited to contribute. Contribution of a chapter will undergo blind peer review to be included subject to recommendation of acceptance by reviewers.

\section{Call for Participation}

What role do people have in the Internet of Things? Compared to the impressive body of research that is currently tackling the technical issues of the Internet of Things, human-centric aspects of agency, engagement, participation, ethics, privacy, are receiving less attention.

The goal of 'Designing the Social Internet of Things' is to contribute to shed light on these aspects. We invite prospective participant to take a humanistic standpoint, explore people's relations with 'things' first, and then build on such relations so as to support socially relevant goals of engagement, relatedness, participation, and creativity.

To participate: Visit the workshop's website. Read the instructions to submit your position paper. Papers will be reviewed by the organizers based on relevance and likelihood to sparkle discussion at the workshop. Note that at least one author of each accepted position paper must attend the workshop and that all participants must register for both the workshop and for at least one day of the conference.

://www.designparticipation.net/2016/11/26/siotchi2017-workshop.html

Before the workshop: contribute to the discussion on Facebook, offer your insights about how you or others use things, in what ways do they enhance people's ives?

At the workshop: bring along a thing (or a placeholder, e.g. a model, a sketch, a picture) that you are keen to discuss in more depth, and be prepared to offer your insights about the contribution of other participants.

After the workshop: you will be invited to contribute a peer-reviewed chapter to the book 'Social Internet of Things' to be published by Springer. 


\section{References}

1. Luigi Atzori, Antonio Iera, and Giacomo Morabito. 2010. The Internet of Things: A survey. Computer Networks 54, 15: 2787-2805.

http://doi.org/10.1016/j.comnet.2010.05.010

2. Margot Brereton. 2013. Habituated Objects: Everyday Tangibles That Foster the Independent Living of an Elderly Woman. interactions 20, 4: 20 24. http://doi.org/10.1145/2486227.2486233

3. Margot Brereton, Alessandro Soro, Kate Vaisutis, and Paul Roe. 2015. The Messaging Kettle: Prototyping Connection over a Distance Between Adult Children and Older Parents. Proceedings of the 33rd Annual ACM Conference on Human Factors in Computing Systems, ACM, 713-716. http://doi.org/10.1145/2702123.2702462

4. Daniel López Gómez. 2015. Little arrangements that matter . Rethinking autonomy-enabling innovations for later life. Technological Forecasting \& Social Change 93: 91-101. http://doi.org/10.1016/j.techfore.2014.02.015

5. Martin Heidegger. 2008. The Origin of the Work of Art. In Basic writings, David Farrell Krell (ed.). HarperCollins Publishers Ltd, New York, NY, 143-212.

6. Chiara Leonardi, Claudio Mennecozzi, Elena Not, et al. 2009. Knocking on Elders' Door: Investigating the Functional and Emotional Geography of Their Domestic Space. Proceedings of the SIGCHI Conference on Human Factors in Computing Systems, ACM, 1703-1712. http://doi.org/10.1145/1518701.1518963
7. Bjorn Nansen, Luke van Ryn, Frank Vetere, Toni Robertson, Margot Brereton, and Paul Douish. 2014. An Internet of Social Things. Proceedings of the 26th Australian Computer-Human Interaction Conference on Designing Futures: The Future of Design, ACM, 87-96.

http://doi.org/10.1145/2686612.2686624

8. Yvonne Rogers. 2006. Moving on from Weiser's Vision of Calm Computing: Engaging UbiComp Experiences. UbiComp 2006: Ubiquitous Computing, Springer Berlin Heidelberg, 404-421. http://doi.org/10.1007/11853565

9. Alessandro Soro, Margot Brereton, and Paul Roe. 2015. The Messaging Kettle: It's IoTea Time. Proceedings of the 5th Decennial Aarhus Conference, 57-59.

10. Alessandro Soro, Margot Brereton, and Paul Roe 2016. Towards an Analysis Framework of Technology Habituation by Older Users. Proceedings of the 2016 ACM Conference on Designing Interactive Systems, ACM, 1021-1033. http://doi.org/10.1145/2901790.2901806

11. Kate Vaisutis, Margot Brereton, Toni Robertson, et al. 2014. Invisible Connections: Investigating Older People's Emotions and Social Relations Around Objects. Proceedings of the SIGCHI Conference on Human Factors in Computing Systems, ACM, 1937-1940.

http://doi.org/10.1145/2556288.2557314 